Strong nonlinear growth of energy coupling during laser irradiation of transparent dielectrics and its significance for laser induced damage

G. Duchateau, M. Feit, S. Demos

August 5, 2011

Journal of Applied Physics 
This document was prepared as an account of work sponsored by an agency of the United States government. Neither the United States government nor Lawrence Livermore National Security, LLC, nor any of their employees makes any warranty, expressed or implied, or assumes any legal liability or responsibility for the accuracy, completeness, or usefulness of any information, apparatus, product, or process disclosed, or represents that its use would not infringe privately owned rights. Reference herein to any specific commercial product, process, or service by trade name, trademark, manufacturer, or otherwise does not necessarily constitute or imply its endorsement, recommendation, or favoring by the United States government or Lawrence Livermore National Security, LLC. The views and opinions of authors expressed herein do not necessarily state or reflect those of the United States government or Lawrence Livermore National Security, LLC, and shall not be used for advertising or product endorsement purposes. 


\title{
Strong nonlinear growth of energy coupling during laser irradiation of transparent dielectrics and its significance for laser induced damage
}

\author{
Guillaume Duchateau ${ }^{1}$, Michael D. Feit ${ }^{2}$, and Stavros G. Demos ${ }^{2}$ \\ ${ }^{1}$ CEA, Centre d'Etudes du Ripault, BP 16, 37260 Monts, France \\ ${ }^{2}$ Lawrence Livermore National Laboratory, 7000 East Avenue, Livermore, CA 94550, USA
}

July 11, 2011

\begin{abstract}
The interaction of high power nanosecond laser pulses with absorbing defects, located in the bulk of transparent dielectric materials and having a multilevel electronic structure, is addressed. The model assumes a moderate localized initial absorption that is strongly enhanced during the laser pulse via excited state absorption and thermally-driven generation of new point defects in surrounding material. This model is applied to laser induced damage in the bulk of potassium dihydrogen phosphate crystals $\left(\mathrm{KH}_{2} \mathrm{PO}_{4}\right.$ or KDP) and plausibly explains how during a fraction of the pulse duration the host material around the defect is transformed into a strong absorber that leads to the sufficiently large energy coupling resulting in a damage event. This scenario is supported by time resolved imaging of material modification during the initial phases of laser induced damage.
\end{abstract}

\section{Introduction}

From tabletop lasers to high power lasers such as NIF or LMJ [1], optical materials (large bandgap dielectrics) are used to transform pulse characteristics such as energy density, pulselength, wavelength, or spatial profile. However, these optical components themselves may suffer from laser-induced damage attributed to absorption by nanoscale defect structures $[2,3,4,5,6]$, especially at $\lambda=351 \mathrm{~nm}(3 \omega)$. The interaction of such absorbing defects with nanosecond laser pulses, leading to sufficient energy deposition to cause a micro-explosion, involving temperatures on the order of $1 \mathrm{eV}$ or larger and pressures on the order of $10 \mathrm{GPa}$, is an active area of research. Since damage is penalizing for the laser facilities, there is a need to understand the physical processes resulting from the interaction of intense laser light with condensed matter. Ultimately, this understanding could lead to technological means of increasing the damage resistance of optical materials.

Within the femtosecond (fs) regime, the physical mechanisms responsible for damage are now well established: multiphoton ionization (MPI) eventually followed by impact ionization leads to a large density of conduction electrons giving rise to optical breakdown [7]. On the fs timescale, with associated intensities generally lying in the $T W / \mathrm{cm}^{2}$ range, the laser pulse interacts via intrinsic processes with matter (eventually with atomic-size defects) while the crystalline lattice is not perturbed [8]. Since the energy transfer time from the electrons to the ions (lattice) is typically a few picosecond (ps), material phase changes and hydrodynamics play a role only subsequent to the energy deposition. 
Within the nanosecond (ns) regime, with intensities lying in the $\mathrm{GW} / \mathrm{cm}^{2}$ range, the situation is dramatically different. Firstly, damage at relatively low laser fluences is initiated by precursor defects whose size may range from a few tens of nanometers (nm) to some hundreds of nm $[2,3,4,5,6]$. Secondly, both thermal conduction and hydrodynamics as well as phase change of material may play a significant role since the laser pulse lasts long enough for these processes to occur. Although plasma formation occurs as in the fs case, a striking difference is that energy deposition is time dependent since heat conduction and hydrodynamics allow energy to be transported away from the small precursor during the laser pulse and influence the properties, such as absorption, of the surrounding material.

The initial steps of ns regime laser damage initiation have drawn attention from a number of researchers in the past attempting to elucidate the nature of the precursor defects and the transformation of the surrounding material. It has generally been concluded $[9,10,11]$ that the initial absorbers are small, submicron in size, that generate plasma that heats and transforms the surrounding material to create additional absorption $[12,13,14]$. The precise mechanism by which modification of the surrounding material can occur has been the subject of a number of studies. It is experimentally known [15] that shockwaves can densify silica and that such densification occurs during laser damage [16]. Molecular dynamics simulations [17] elucidated structural changes that occur in fused silica at the expected shock induced pressures and suggested associated relevant electronic structure modifications might also occur. Previous work $[9,10]$ also pointed out the importance of temperature dependent absorption in the damage initiation process. Early experimental studies of the temperature dependence of absorption [18] suggested at least a partial bandgap collapse with increasing temperature in fused silica, analogous to the complete bandgap collapse known for semiconductors [19]. An experimental demonstration of reduced damage threshold in fused silica with temperature was found in [14]. More recently, observation of induced absorption in glasses due to ultrashort laser pulse exposure has also been reported [20]. Additionally, recent density-functional theory calculations [21] have shown that silica absorption spectra are expected to exhibit exponential tails near the band edge at elevated tempertatures.

The basic concept of a laser driven moving absorption wave fed by either heat conduction or radiation from hot plasma has been extensively developed [22], initially in fluids and later in solids, e.g. in studies of slow moving combustion waves and supersonic detonation waves. Laser induced absorption waves [23] in transparent material such as air are well known. Laser damage in a nominally transparent material allows creation of an absorption wave if the transparent material becomes absorbing when it is heated. This was predicted theoretically [9] before it was observed experimentally [24]. Early experiments found the absorption wave moves at subsonic velocity which varies roughly as the square root of laser intensity.

In potassium dihydrogen phosphate $\left(\mathrm{KH}_{2} \mathrm{PO}_{4}\right.$ or $\left.\mathrm{KDP}\right)$, the plasma state is assumed to be created in a time much shorter than the pulse duration $[2,5]$. Under these conditions, the defect absorption was assumed to be constant in space and time. Nevertheless, the first detailed information was recently provided regarding KDP bulk damage where information about the electronic structure of the cluster of atomic defects (damage precursor) and the corresponding transition rates were extracted [25]. Based on these results, a time-dependent model can be developed for the description of the energy absorption (in space and time) during the damage initiation event in this material.

In the present work, a new mechanism based on thermally-driven secondary point defect formation (SPDF) in the precursor and the surrounding material is introduced to model the modification of absorption where the essential physical mechanisms are taken into consideration in a self consistent way: temperature- 
dependent interband transitions and heat transfer equations are coupled to predict the spacetime evolution of the energy absorption and temperature. Although, as noted above, the idea of temperature induced absorption is not new and, for example, Ref. [14] suggests the possibility of defect generation as a mechanism, the present work is the first systematic quantitative development of this idea combined with an experimentally motivated electronic structure model [25] to provide a detailed description. Based on this approach, it is shown how damage initiation may occur because of a strong nonlinear growth of energy coupling. In particular, the formation of an absorption front (AF), moving at a speed one order of magnitude slower than the sound speed, is predicted, consistent with experiments [24] and other models [21]. This mechanism is essentially expected at the beginning of the interaction where the material undergoes major change of its electronic, optical and structural properties. Experimental results using time resolved imaging of material modification during the initial phases of laser induced damage in the bulk of fused silica are suggestive that the model may be more general, also describing similar processes in other optical materials with appropriate modifications for each material. Hereafter we refer to the precursor with electronic structure (PES) model augmented with secondary point defect formation (SPDF) as the PES-SPDF model.

\section{Theoretical model}

Assume for simplicity that damage originates from absorption by a spherical precursor defect whose radius is set to $100 \mathrm{~nm}[2,5]$. This precursor defect is assumed to be a cluster of point defects with initial density $n_{0}=5 . \times 10^{19} \mathrm{~cm}^{-3}[25]$, the latter introducing excited states located in the band gap [25, 26]. A schematic illustration of the precursor band structure is given by Fig. 1 where, at $3 \omega, 3$ photons are required to promote the valence electrons to the conduction band. Due to these excited states (ES), a valence electron passes through the ES's before reaching the conduction band: three sequentiel one-photon absorption processes can bridge the band gap. Taking electron and ion temperatures equal, the lattice heating is assumed to be due to non-radiative relaxation of electrons populating the ES's and inverse Bremstrahlung. At $3 \omega$, the rate equations governing the evolution of the electron densities are the following [25]:

$$
\left\{\begin{array}{l}
\frac{\partial n_{c b}}{\partial t}=\sigma_{1}^{\prime} F_{p} n_{2}-\frac{n_{c b}}{\tau_{3}} \\
\frac{\partial n_{2}}{\partial t}=\sigma_{1} F_{p} n_{1}-\sigma_{1}^{\prime} F_{p} n_{2}-\frac{n_{2}}{\tau_{2}}+\frac{n_{c b}}{\tau_{3}} \\
\frac{\partial n_{1}}{\partial t}=\sigma_{1} F_{p} n_{d}-\sigma_{1} F_{p} n_{1}-\frac{n_{1}}{\tau_{1}}+\frac{n_{2}}{\tau_{2}} \\
\frac{\partial n_{d}}{\partial t}=-\sigma_{1} F_{p} n_{d}+\frac{n_{1}}{\tau_{1}}
\end{array}\right.
$$

where $F_{p}$ is the photon flux given by $I / \hbar \omega$ with $I$ the laser intensity. Here we consider a flat-in-time pulse, i.e. $I(t)=I_{0} \cdot \sigma_{1}$ and $\sigma_{1}^{\prime}$ are one-photon-absorption cross sections. $n_{i}$ is an electronic density where $i$ stands for the valence band (VB), an ES, or the conduction band $(\mathrm{CB}) . n_{d}$ is the density of valence electrons that can be promoted to the ES's due to point defects. $\tau_{1}, \tau_{2}$, and $\tau_{3}$ are non-radiative relaxation times for state ES1, ES2, and the conduction state, respectively.

This model reproduced experimental trends in the case where two wavelengths are present and has been used to obtain best estimate values of the absorption cross sections between the various states, and density of atomic defects within each defect cluster acting as damage precursor [25]. This model provides the foundation to obtain insight, for the first time, into the early material transformation and behavior as a result of high power laser excitation that leads to a strong coupling of the laser light into localized areas of the material and subsequent laser induced damage. This can be achieved by including in the model the energy balance in the affected material volume of all major mechanisms involved in the process and monitor the 
dynamics under realistic excitation conditions. The first important mechanism is heat diffusion from the initially absorbing volume (precursor) into the surrounding host material which is known to significantly influence the temperature evolution in the nanosecond timescale. The heating of conduction electrons by inverse Bremstrahlung should also be taken into account at later stages of the excitation process and so has been introduced into the Fourier heat equation. Finally, the heating of the material is accompanied by the generation of new point defects arising from the breaking of bonds. It follows that heat diffusion may induce new point defects not only in the precursor, but also in the surrounding host material leading to an expansion of the absorbing zone. In the case of KDP crystals, the energy cost of breaking hydrogen bonds, leading to hydrogen defects such as interstitials and vacancies, is very low. It is assumed that the breaking of hydrogen bonds also induces states located in the band gap [27] and generation of additional defects with complex electronic structure (similar to that used in this model) as it has been shown for the case for x-ray irradiation of the material by Chirila et al [28]. For simplicity, we describe the new defects with the same rate equations as the precursor to treat the evolution of the electronic populations and their contribution to the lattice heating. Within this new framework, the system which has to be solved is now the following:

$$
\left\{\begin{array}{l}
\frac{\partial n_{c b}}{\partial t}=\sigma_{1}^{\prime} F_{p} n_{2}-\frac{n_{c b}}{\tau_{3}} \\
\frac{\partial n_{2}}{\partial t}=\sigma_{1} F_{p} n_{1}-\sigma_{1}^{\prime} F_{p} n_{2}-\frac{n_{2}}{\tau_{2}}+\frac{n_{c b}}{\tau_{3}} \\
\frac{\partial n_{1}}{\partial t}=\sigma_{1} F_{p} n_{d}-\sigma_{1} F_{p} n_{1}-\frac{n_{1}}{\tau_{1}}+\frac{n_{2}}{\tau_{2}} \\
\frac{\partial n_{d}}{\partial t}=-\sigma_{1} F_{p} n_{d}+\frac{n_{1}}{\tau_{1}}+\frac{\partial N_{d}}{\partial t} \\
C \frac{\partial T}{\partial t}=\nabla(\kappa \nabla T)+\left(n_{1} E_{01} / \tau_{1}+n_{2} E_{12} / \tau_{2}+n_{c b} E_{23} / \tau_{3}-E_{a} \frac{\partial N_{d}}{\partial t}+S_{i B} n_{c b}\right)
\end{array}\right.
$$

where notations for the rate equations are similar to those of Eq. (1). $E_{01}, E_{12}$, and $E_{23}$ are the energy differences between the valence band and ES1, ES1 and ES2, and, ES2 and the bottom of the conduction band, respectively [25]. $\kappa$ and $C$ are the thermal conductivity and the heat capacity, respectively. Assuming that $E_{a}$ is the energy required to form a new point defect, the associated term in Eq. (2) reads $-E_{a} \partial N_{d} / \partial t$ where $N_{d}$ is the density of point defects. It is reasonable to think that $N_{d}$ follows an Arrhenius law, thus reading:

$$
\left\{\begin{array}{l}
N_{d}(r, t)=n_{0}+K_{0} \exp \left(-\frac{E_{a}}{k T(r, t)}\right) \text { inside the precursor defect } \\
N_{d}(r, t)=K_{0} \exp \left(-\frac{E_{a}}{k T(r, t)}\right) \text { outside the precursor defect }
\end{array}\right.
$$

where $K_{0}$ is a constant which is estimated hereafter. Note that a point defect provides one valence electron which may be excited to higher states in Eq. (2). $S_{i B}$ describes the heating of conduction electrons by inverse Bremstrahlung. Within the Drude model, it is proportional to the electronic conductivity and is given by:

$$
S_{i B}=\frac{e^{2} \hbar \omega F_{p}}{8 m c \varepsilon_{0}\left(\omega^{2}+\nu_{c}^{2}\right)} \nu_{c}
$$

where $\nu_{c}$ is the collisional frequency.

The thermal conductivity appearing in the heat transfer equation of system (2) can be written as $\kappa(r, t)=\kappa_{i}+\kappa_{e}(r, t)$ where $\kappa_{i}$ and $\kappa_{e}$ are contributions supported by ions and electrons, respectively. For KDP, $\kappa_{i}=1.3 \mathrm{~W} / \mathrm{m} / \mathrm{K}$ and we determine the electronic thermal conductivity assuming that the ionized KDP has a metallic behavior. We thus use the Wiedemann-Franz law:

$$
\frac{\kappa_{e}}{\sigma}=\frac{3}{2}\left(\frac{k_{B}}{e}\right)^{2} T
$$


where $\sigma$ is the electrical conductivity assumed to be $n_{c b} e^{2} \nu_{c} / m\left(\omega^{2}+\nu_{c}^{2}\right)$ within the Drude model. The electronic thermal conductivity thus reads:

$$
\kappa_{e}=\frac{3}{2} k_{B}^{2} \frac{n_{c b} T \nu_{c}}{m\left(\omega^{2}+\nu_{c}^{2}\right)}
$$

This model also incorporates the following assumptions:

a) The non-radiative relaxation of conduction electrons does not depend on their kinetic energy. Alternatively, we could introduce supplementary rate equations [29]. The latter formalism also allows including impact ionization. Nevertheless, at $3 \omega$ with relatively low intensities similar to those found in currently available laser system or near future designs, avalanche may not be significant [7].

b) The electrons are in thermal equilibrium with the lattice.

c) We do not introduce a diffusion operator on the electronic temperature. In other words, we assume for the moment $\tau_{e-i o n} \ll \tau_{e-e}$ since the electronic and ionic temperatures are assumed to be equal. The latter assumption is based on the fact that the inelastic interaction between the electrons and the ions takes place on the ps time scale while the energy transport processes described in this model occur on a much slower time scale.

d) The thermal parameters (heat capacity and ionic thermal diffusivity) are assumed temperature independent, equal to those of KDP at room temperature.

Within our model, each physical quantity varies in space and time. System (2) is solved numerically with a finite difference scheme in spherical coordinates where the spatial step has been set to $1 \mathrm{~nm}$ and the time step is adapted to ensure the algorithm convergence. The initial conditions are the following:

$$
\begin{aligned}
& \text { In the precursor defect }(r \leq 100 \mathrm{~nm}):\left\{\begin{array}{l}
n_{c b}=0 \\
n_{2}=0 \\
n_{1}=0 \\
n_{d}=n_{0} \\
T=T_{0}
\end{array}\right. \\
& \text { Outside the precursor defect: }\left\{\begin{array}{l}
n_{c b}=0 \\
n_{2}=0 \\
n_{1}=0 \\
n_{d}=0 \\
T=T_{0}
\end{array}\right.
\end{aligned}
$$

Finally, we have to choose a criterion for describing the absorption front (AF), and subsequently to evaluate the area of the absorbing zone with respect to time. Here we assume that the local absorption is proportional to the instantaneous point defect density while the radius of the AF is determined by the distance from the center of the precursor where a given point defect density has been reached Also, we assume that the illuminated area is significantly absorbing if $N_{d} \geq n_{0}$.

\section{Modeling results and discussion}

The value of the modeling parameters are listed in Table 1 unless otherwise explicitly stated. Also, the laser parameters have been set to values relevant to the operational conditions of ICF class laser systems such as NIF or LMJ [1]. Regarding the parameters governing the electronic densities evolution (one-photon absorption cross section, non-radiative relaxation time, and energy difference between adjacent ES's), the values are those of [25] which were extracted by fitting experimental damage-testing data under simultaneous excitation at two wavelengths. The collisional time has been set in the fs range in accordance with current knowledge [7], i.e. $\tau_{c}=1 \mathrm{fs}$. The initial point defect density within the defect cluster (damage 
Table 1: value of the parameters

\begin{tabular}{lll}
\hline Description of variables and parameters & Symbol & Value \\
\hline Photon energy & $\hbar \omega$ & $3.54 \mathrm{eV}$ \\
Laser intensity & $I$ & $3 \mathrm{GW} / \mathrm{cm}^{2}$ \\
\hline one-photon absorption cross section & $\sigma_{1}$ & $1.5 \times 10^{-18} \mathrm{~cm}^{2}$ \\
one-photon absorption cross section & $\sigma_{1}^{\prime}$ & $7 \sigma_{1}$ \\
Recombination time & $\tau_{1}$ & $1 \mathrm{~ns}$ \\
Recombination time & $\tau_{2}$ & $50 \mathrm{ps}$ \\
Recombination time & $\tau_{3}$ & $1 \mathrm{ps}$ \\
Energy gap between ground state and level 1 & $E_{01}$ & 2.3 \\
Energy gap between level 1 and level 2 & $E_{12}$ & 3.2 \\
Energy gap between level 2 and CB & $E_{23}$ & 2.2 \\
Collisional frequency & $\nu_{c}$ & $10^{15} \mathrm{~s}^{-1}$ \\
\hline Heat capacity & $C$ & $2 \mathrm{~J} / \mathrm{K} / \mathrm{cm}^{3}$ \\
Thermal diffusivity of DKDP & $D_{i}$ & $10^{-6} \mathrm{~m}^{2} / \mathrm{s}$ \\
Room temperature & $T_{0}$ & $300 \mathrm{~K}$ \\
\hline Initial point defect density & $n_{0}$ & $5 \times 10^{19} \mathrm{~cm}^{-3}$ \\
Maximum point defect density & $K_{0}$ & $10^{22} \mathrm{~cm}^{-3}$ \\
Energy of a hydrogen bond in KDP & $E_{a}$ & $0.042 \mathrm{eV}$ \\
Radius of the precursor defect & $r_{0}$ & $100 \mathrm{~nm}$ \\
\hline
\end{tabular}

precursor) is the one of [25]. Note that the latter values provide the best agreement with experimental data, but slight deviations from these values also render it possible to predict correct trends. Furthermore, it is assumed that the new defects arise from the breaking of hydrogen bonds which are the weaker bonds in this material. Since the primitive cell of KDP consists of two units of $\mathrm{KH}_{2} \mathrm{PO}_{4}$ with a volume close to $360 \AA^{3}$, the maximum point defect density corresponds roughly to four interstitial hydrogen atoms in the latter volume, i.e. $K_{0} \simeq 10^{22} \mathrm{~cm}^{-3}$. The energy, $E_{a}$, required to break a hydrogen bond provided by [30] is used. It is noteworthy that we have studied the influence of this parameter on the value of the AF velocity. As expected, the lower $E_{a}$, the easier the SPDF, and the faster the speed of the AF. It will be shown hereafter that the present approach is able to mimic the experimental results for the lowest, physically reasonable, value of $E_{a}$. We thus have set $E_{a}$ to the measured value $0.042 \mathrm{eV}$ [30] compatible with the existence of a centre with this activation energy.

Fig. 2 exhibits the predicted lattice temperature, the electronic densities, and the absorbed power density as a function of time in the center of the precursor $(r=0)$. The temperature can reach a few $10000 K$ at the end of the pulse, but this model is not intended to describe the material state at these conditions. Specifically, we hypothesize that the present approach is valid for lower temperatures, possibly as long as the temperature does not exceed $10000 \mathrm{~K}$, i.e. the temperature for which the band gap collapse engages significantly [31]. Within the present parameters, this corresponds to a time shorter than roughly $1 \mathrm{~ns}$, which indicates the time interval during the early phase of damage initiation where the PES-SPDF model may accurately describe the processes taking place. Despite the crudity of this approach for times longer than $1 \mathrm{~ns}$, it is worth noting that the predicted behaviors have been considered hereafter up to 6 ns in order to shed light on the energy coupling occurring as long as the pulse is switched on. Actually, the predicted trends should be correct since the influence of a band gap collapse is equivalent to 
enhance the absorption cross section in the present approach.

As expected, the prediction of a fast temperature rise is due to the increase in absorption related to the point defect density increase (that is a function of the temperature). Also, the shape of $T(t)$ clearly exhibits a non-linear behavior: a thermal explosion occurs at $t \simeq 0.5 \mathrm{~ns}$. This time corresponds to a temperature close to $500 \mathrm{~K}$, i.e. close to $0.042 \mathrm{eV}\left(=E_{a}\right)$ for which the SPDF becomes significant. After the termination of the laser pulse, the relative decrease in temperature of the affected volume is slower than the one predicted by previous models $[2,5]$. This is due to the fact that the heated region is significantly larger when including SPDF. Indeed, as shown by Fig. 3, the new radius is close to $250 \mathrm{~nm}$, resulting in a smaller surface to volume ratio and outgoing heat flux. This size evolution is discussed in more details hereafter.

As shown by Fig. 2(b), the thermal explosion is due to the fact that ES1, ES2 and the CB state quickly reach near stationary high densities in excess of $10^{20} \mathrm{~cm}^{-3}$ (whose non-radiative relaxation induce the increase in the temperature of the lattice). Despite the initially low point defect density, these high densities are due to the SPDF as shown by the orange curve of Fig. 2(b) showing that the defect density evolves from $5 \times 10^{19} \mathrm{~cm}^{-3}$ to the maximum value $10^{22} \mathrm{~cm}^{-3}$. Accordingly, the density of valence electrons able to be easily excited $\left(n_{d}\right.$, black curve of Fig. 2(d)) can increase whereas transitions from the valence band to ES1 would induce the opposite behavior (depopulation) as usually observed without SPDF.

The absorbed power density, leading to the temperature rise, is shown by Fig. 2(c) as predicted by the PES-SPDF approach. It appears that the power density associated with the SPDF, which acts as an energy loss mechanism, is negligible compared to that transferred from the electrons to the lattice due to their nonradiative relaxation. That is notably due to the fact that $E_{a} \simeq E_{i j} / 10$. It also appears that the inverse Bremstrahlung does not contribute significantly to temperature variations due to its low absorbed power density. This is mainly due to the relatively small density of conduction electrons $\left(\simeq 10^{20} \mathrm{~cm}^{-3}\right)$ compared to the critical plasma density close to $10^{22} \mathrm{~cm}^{-3}$ at $3 \omega$. Actually, the results suggest that the non-radiative relaxation of the conduction electrons is the main contributor to the temperature rise, followed by the electrons in ES2 and ES1. The contribution of a given state, labeled $i$, is mainly governed by the term $n_{i} E_{i j} / \tau_{i}$ with $j=i+1$. Since the energy differences between two adjacent states is similar in the present system under considerations, the only factor $n_{i} / \tau_{i}$ allows one to compare the various contributions. Consequently, despite that the conduction electrons have the lowest density, their short non-radiative relaxation time makes them the largest contributors to the energy deposition and subsequent temperature rise.

The spatial evolution of the lattice temperature, the electronic densities, and the absorbed power density at $t=1 n s$ is depicted on Fig. 3. Regarding the temperature, it appears that the extent of the heated region is significantly larger than the standard length of thermal diffusion close to $110 \mathrm{~nm}$ for which a significant temperature rise would occur. Let us define the extent as the region for which the temperature is larger than $500 \mathrm{~K}$, this temperature corresponding to a significant phase transition or SPDF. From Fig. 3(a), it appears that the heated region extends to roughly $250 \mathrm{~nm}$. This behavior is a first indication regarding the evolution of the size of the absorbing zone outside the initial precursor volume with respect to time. This behavior is also observed when considering the spatial evolution of the electronic densities and the according absorbed power density, as shown by Figs. 3(b) and 3(c). Significant electronic densities and absorbed power density can be observed up to $250 \mathrm{~nm}$ or so, confirming the absorbing zone has grown with respect to time since it only occurs in the volume $r<r_{0}(=100 \mathrm{~nm})$ initially.

Within the framework of this model, the physical quantities depend both on space and time. In order to understand the origin of these variations, the evolution 
of the temperature and the density of conduction electrons has been plotted in Figs. 4 and 5. Fig. 4 shows that a significant temperature rise occurs in a continuously increasing volume with a speed of expansion that seems to be almost constant since the iso-temperature lines for $r>100 \mathrm{~nm}$ are close to a straight line (when assuming flat-in-time pulse). The absorption associated with the electronic densities confirms this trend: the iso-density lines of the conduction band electrons as a function of space and time shown in Fig. 5 indicate that the plasma is expanding at an almost constant rate.

The expansion of the absorbing front is associated with the generation of new point defects as explained in the previous section (criterion $N_{d}>n_{0}$ ). The evolution of the AF as a function of time is plotted in Fig. 6. Note we have checked that other criteria such that $T>T_{c}$ or $n_{c b}>n_{c}$ provide very similar trends. As expected, the shape of the curve is similar to an iso-density line of Fig. 5. With the onset of $\mathrm{AF}$ expansion, its position (radius $r_{A F}$ ) according to this model evolves almost linearly with time. In comparison, a purely diffusive process would lead to an evolution that is closer to a square root behavior, for which $l=2 \sqrt{D t}$ where $l$ is the characteristic spatial scale, i.e. the thermal length of diffusion, and $D$ is the thermal diffusivity. However, the nonlinear processes described above involving the coupling between $N_{d}, T$, and the absorbed power density lead to a behavior departing from the standard square root law. To better evaluate the importance of introducing thermally-driven SPDF in this model, the pure diffusive process is also considered within the range $t \in[0 ; 6 \mathrm{~ns}]$, where we have performed a fit of the numerical results with the function $r_{A F}=100 \mathrm{~nm}+2 \sqrt{D t}$ where $D$ is the fitting parameter. The best fit is obtained with $D=15 \times 10^{-6} \mathrm{~m}^{2} / \mathrm{s}=15 D_{i}$. The resulting curve is the red one of Fig. 6.

This result shows that the introduction of SPDF leads to an increased energy deposition process allowing the AF to spatially expand with time. The instantaneous speed of expansion of the radius of the absorbing volume is simply given by $v_{A F}=\partial r_{A F} / \partial t$. The inset of Fig. 6 shows the evolution of this quantity as a function of time. Soon after the pulse is switched on, it appears that $v_{A F}$ tends to a constant close to $170 \mathrm{~nm} / \mathrm{ns}$ within the present excitation conditions. Actually, this value is reached after roughly $1 \mathrm{~ns}$. This time corresponds to the saturation of the electronic densities, i.e. when a stationary regime is established. Note that the fluctuations of $v_{A F}$ are due to the numerical discretization of the problem.

Fig. 7 shows the evolution of the average speed of expansion as a function of the laser intensity. It is defined as:

$$
<v_{A F}>_{t}=\frac{1}{\tau} \int_{0}^{\tau} d t v_{A F}(t)=\frac{r_{A F}(\tau)-r_{0}}{\tau}
$$

It appears that the higher the intensity, the faster the speed of expansion of the AF although it grows less than linearly with intensity. See Eq. (12) below for insight as to the origin of this type of behavior. More precisely, a fit based on a power law leads to $v_{A F} \simeq 79 . I^{0.6}$ where $v_{A F}$ and $I$ are in units of $\mathrm{nm} / \mathrm{ns}$ and $G W / \mathrm{cm}^{2}$ respectively.

From these studies, it appears that the speed of expansion of the absorbing front during the early phase of damage initiation can reach a few hundreds of $\mathrm{nm} / \mathrm{ns}$ in operationally relevant conditions of laser excitation. When the precursor defect and its vicinity are heated up to a temperature of a few $10000 \mathrm{~K}$, a significant pressure rise is expected while band gap collapse may occur at even lower temperatures. However, hydrodynamic considerations may be important during the evolution of the processes described in this model. Specifically, material displacements due to increase of the pressure arising from the increase of the temperature are expected in the vicinity of the precursor defect $[32,33,34]$. An evaluation of these material displacements can be performed within reasonable assumptions. For a constant 
absorbed power density $W$, the speed of expansion due to hydrodynamics reads (see Appendix A):

$$
v_{H}=\frac{\Gamma W r_{0}}{\rho c_{0}^{2}}
$$

Within the present framework, the absorbed power density $\mathrm{W}$ results from the nonradiative relaxation of several states. It can thus be written as: $W=\sum_{i} W_{i}$ where $i$ corresponds to ES1, ES2, and the conduction band. Note that this velocity is a function of the laser intensity through the absorbed power.

Both influences should be taken into consideration in order to evaluate the speed of expansion of the absorbing front. Actually, we can assume that the SPDF occurs in a material moving at constant velocity. Under this assumption, all the developments of Sec. 2 remain unchanged assuming that the densities do not significantly vary due to volume changes. It follows that the speed of expansion of the absorbing front can be approximated by:

$$
v_{A F}=v_{S P D F}+v_{H}
$$

where $v_{S P D F}$ and $v_{H}$ are $A F$ velocities induced by SPDF and hydrodynamics, respectively. Within standard conditions of high power laser for which $F \simeq 10 \mathrm{~J} / \mathrm{cm}^{2}$ and $\tau=3 \mathrm{~ns}\left(I \simeq 3 \mathrm{GW} / \mathrm{cm}^{2}\right)$, calculations lead to $v_{S P D F}=170 \mathrm{~nm} / \mathrm{ns}$ and $v_{H}=171 \mathrm{~nm} / \mathrm{ns}\left(W=5 . \times 10^{13} \mathrm{~W} / \mathrm{cm}^{3}\right.$, see Fig. 2), leading to $v_{A F}=341 \mathrm{~nm} / \mathrm{ns}$.

\section{Time resolved imaging of damage initiation}

Within the model of the damage initiation mechanism in KDP presented in the previous section, direct imaging of the very early processes leading to the build up of the localized absorption of the material would be impossible due to the small size of the precursors and the spatial scale of the effects. Imaging in KDP is further complicated by optical birefringence effects. However, one can postulate that the processes discussed in this work are not specific to KDP but more general, applying even to materials that do not exhibit the highly localized damage behavior within even small volumes at lower fluence of KDP. If we assume that the damage precursors in the material are, unlike the case in KDP, nearly uniformly distributed over small volumes in the bulk [35], the damage initiation would reflect the local laser beam intensity and fluence as well as the local defect density [36, 37]. Consequently, if the pulses of a laser beam were focused in such material, manifestation of the localized energy deposition would first take place at the focal region of the laser beam where the highest intensity exists. Such damage behavior is exhibited by fused silica, which represents another optical material of great importance in large aperture laser systems. The damage behavior in the bulk of fused silica has been discussed elsewhere [38] but the initial behavior of the material at the early stages of damage initiation was not resolved. To measure how the absorption is building up at the focal region of the material requires higher temporal resolution than that used in [38] in order to capture images very early in the process. Based on this work, before the material reaches the point of bandgap collapse accompanied by a fast expansion of the absorbing region [31] and launch of a shockwave, there should be a time period where the absorption is building up from zero to a very high value. To test this concept, we utilized a time-resolved microscope system that provides adequate temporal resolution and has recently been described elsewhere [39]. In brief, two Q-switched Nd:YAG laser systems were synchronized using an electronic pulse delay generator. The first laser (pump) was operated at $355 \mathrm{~nm}$ while each pulse had 3-ns time duration (at full-width-half-maximum, FWHM). Its output beam was focused with a radius $\left(1 / e^{2}\right)$ of $5 \pm 1 \mu \mathrm{m}$ within the bulk of the material $(1 \mathrm{~cm}$ thick fused silica substrates) with a beam depth of focus of $200-230 \mu m$ which is consistent 
with the observed length of the damaged region [38]. The energy was $535 \pm 50 \mu \mathrm{J}$, corresponding to intensities up to $230-250 \mathrm{GW} / \mathrm{cm}^{2}$.

The time-resolved microscopy system was configured for these experiments in the trans-illumination (shadowgraphy) imaging configuration utilizing two cameras equipped with polarization elements to record the two orthogonal polarization image components. Time-resolved images of the transient material behavior was enabled by strobe light illumination provided by the second laser that was operated at 532 $\mathrm{nm}$ with $150 \mathrm{ps}$ pulse time duration (FWHM). The linearly polarized output of this laser was used to illuminate the area at the focal point of the pump beam and the surrounding volume. A polarizing cube beam splitter positioned after the imaging optics of the microscope separated the two polarization image components which were subsequently recorded at predetermined delays using two CCD array detectors. In this configuration, the orthogonal polarization image component captures the location where stress causes rotation of the polarization of the illumination beam, thus proving a method to capture the spatial distribution of transient (and residual) stress fields. The parallel polarization component captures the loss of transmission by the object (damage site) arising from absorption and/or scattering. The time delays between pump and probe pulses were measured from each recorded damage event from their peak intensities.

Fig. 8 shows transient images obtained during the early stages of damage initiation. Each image represents a different damage event but, due to the consistent repeatability of the observations as a function of delay time, they accurately represent the material behavior as a function of delay. The images on the left side (aI, bI, cI) represent the parallel polarization image components while the images shown on the right side (aII, bII, cII) represent the corresponding orthogonal polarization image components. The pump laser beam traversed left-to-right at about 30 degrees with respect to the image plane and the image focus was maintained at the tip of the forming site (left portion of image is slightly out of focus).

The time of first observation of material modification at the region where the pump beam is focused was observed at about -2.40 ns delay under the excitation conditions described above. The image (aI) shown in Fig. 8 was obtained at -2.20 ns delay and is associated with a transmission loss at the center of the modified region of about $10 \%$. This indicates a change of the localized complex index of refraction leading to defocusing of the transmitted probe beam and/or loss of energy via absorption. This transmission loss continues to increase for longer delays until it reaches the detection limit of our system (at about 98\%) and delay of about -1.60 ns. An example of this case is shown in image (bI) obtained at a delay of $-1.59 \mathrm{~ns}$. Immediately after this time delay, the launching of a shock wave and fast expansion of the absorbing region are observed. The effects observed after this point will be discussed elsewhere. The image (cI) obtained at $0.30 \mathrm{~ns}$ delay is used as an example of the appearance of the shock front using this imaging system. From these images it is evident that there is no shock front present in the images (aI) and (bI) obtained at earlier delays.

Examination of the cross polarized image components at the corresponding delay times shown in images (aII), (bII) and, (cII) demonstrate that there is stress building up even at the case of -2.20 ns delay. These images are individually contrastenhanced to capture the presence of a localized depolarization of the probe light and show the areas where most depolarization takes place. After the launch of the shock wave, the region that seems to cause most depolarization is just behind the shock-front as shown in image (cII). However, at earlier delays, the region that provides most depolarization is around the focal region of the pump beam.

The energy deposited in the material though the absorption of laser light instigates a rise in the localized temperature. As the temperature increases, the material expands and the surrounding cold material begins to be exposed to a pressure and 
to heat conduction. The area where depolarization is observed in image (aII) corresponds to the volume surrounding the focal region, providing evidence that this prediction of the model may be valid. The image shown in (bII) obtained near the end of this early phase (-1.59 ns delay) shows that the depolarization effect has increased and is now visible even in the out of focus region of the image (left), indicative that the stress continues to increase during this time period. In addition, within the resolution of this system (on the order of $1 \mu \mathrm{m}$ ), no significant expansion of the stressed region around the focal point is observed between the $-2.2 \mathrm{~ns}$ and -1.59 ns delays. This indicates that the expansion speed of this absorbing region is lower that the resolution limit of our system (on the order of $1 \mathrm{~km} / \mathrm{s}=1000 \mathrm{~nm} / \mathrm{ns}$ ). It must be noted that the speed of sound in fused silica is $5.9 \mathrm{~km} / \mathrm{s}$.

There are two possible ways material distant from the original absorption site can be heated to increase absorption. One way is via heat conduction, similar to a combustion wave or deflagration, in which the absorption front is expected to travel at less than the sound velocity. The second way is via shockwave heating, similar to a detonation wave, in which the absorption front is expected to travel at greater than the sound velocity. Since the observation is of subsonic velocity, we suppose a combustion wave, i.e. a comparatively weak shockwave.

We can obtain an idea of how the absorption front velocity should depend on laser intensity as follows. We suppose absorbing defects are created via a temperature dependent reaction rate $\Gamma(T)=p \exp \left(-E_{a} / k T\right)=1 / \tau(T)$ which also defines the typical reaction time $\tau$. The rate is characterized by pre-exponential factor $p$ and activation energy $E_{a}$. The temperature that a small precursor of radius $b$ reaches during exposure to laser intensity $I$ is given by [11]:

$$
T=\alpha \frac{b I}{4 \kappa}
$$

where $\kappa$ is the thermal conductivity and $\alpha$ is the fraction of incident light absorbed. The position $\delta$ of the moving absorption front will be given by an expression of form [22] $\delta=C \sqrt{D \tau}$ where $D$ is the thermal diffusivity and $C$ is a coefficient which may be large. The absorption front velocity is then given by $v_{A F}=\delta / \tau=C \sqrt{D \Gamma}$. Putting these three ingredients together, we find the front velocity to be given by:

$$
v_{A F}=C \sqrt{D p} \exp \left(-\frac{\eta}{I}\right) \quad ; \text { where } \eta=\frac{2 \kappa E_{a}}{\alpha b k}
$$

and to depend exponentially on the inverse of the laser intensity. The velocity is expected to be subsonic and to increase slowly with increasing laser intensity. This is consistent with both the experiments and the detailed numerical calculations reported here. We note that the simulations of [31] indicated a higher absorption front velocity would occur if temperature dependent thermal properties were accounted for. That has not been done here.

The experiments suggest that the time interval between the onset of observation of absorption near the focal region to the time point of the launch of the shock wave is about $0.8 \mathrm{~ns}$. During this time, the intensity of the pump pulse is continuously increasing (effect is observed at negative delays, before the peak of the pump pulse). Therefore, it is not possible to extract some information on its dependence on laser intensity but it clearly shows that it is on the order of $1 \mathrm{~ns}$ under the excitation parameters used in this experiment. It is expected [11] on general grounds, that small precursors will lead to a plasma fireball (free electrons) that can grow rapidly up to a size comparable to an optical wavelength. At this point, volume absorption of laser energy in the plasma becomes surface absorption and the fireball growth becomes much slower. 


\section{Concluding remarks and outlook}

Compared to the femtosecond regime, the particularity of the nanosecond regime is that thermal conduction, hydrodynamics and subsequent material properties evolution can occur within the same timescale as the pulse duration. The latter coupling has been modeled with in particular a thermally-driven absorption modification based on point defect formation. This approach has rendered it possible to explain the damage initiation in the nanosecond regime, indicating in particular that damage results from a strong nonlinear energy coupling. Further, this approach accounts for the time evolution of the absorbing area as experimentally observed. Its velocity is found to be one order of magnitude smaller than the speed of sound for standard laser illumination conditions. Furthermore, this work suggests that the above-mentioned energy coupling scenario may be a general feature in common to optical materials. Despite having briefly studied the influence of hydrodynamics on the absorption front velocity, a full coupling of hydrodynamics with the present model is planned in the future to remove some assumptions and increase the accuracy of the predictions.

This work helps to establish a new baseline regarding the required optical properties of impurities or defect clusters embedded in optical materials capable of producing sufficient amount of energy deposition to cause laser induced damage. It has been postulated that in order to initiate laser damage by a small (sub-micrometer in diameter) precursor, the absorption coefficient must be significantly larger than the bulk one. This work demonstrates that defects with electronic structure and linear absorption cross sections commonly found in various defects and impurities in transparent dielectrics have the potential upon laser excitation to initiate a cascade process that can subsequently lead to a laser induced micro-explosion (damage).

\section{Acknowledgments}

We want to thank R. A. Negres, R. Raman, L. Hallo, D. Hébert, and V. Tikhonchuk for helpful discussions. This work was performed in part with support by CEA (France). This work was performed in part under the auspices of the U.S. Department of Energy by Lawrence Livermore National Laboratory under contract DE-AC52-07NA27344.

\section{A Evaluation of the expansion of the absorbing area due to hydrodynamic phenomena}

In order to evaluate the speed of expansion of the plasma due to the hydrodynamic influence, the following derivation is proposed. One can assume that since the plasma is significantly heated, a pressure wave is generated. The associated rarefaction wave creates a void whose size increases with respect to time [32, 40, 34]. The cavity expansion pushes the surrounding matter leading to the so-called plasma expansion. The corresponding velocity is roughly given by the speed of expansion of the cavity, the latter being defined during the acoustic timescale for which the rarefaction wave forms the void. Note that after the acoustic timescale, the pressure wave escapes the initial zone where the energy is deposited (precursor defect). The pressure inside the absorbing zone thus drops and even if the plasma can continue to absorb, the hydrodynamic contribution to its expansion (due to pressure gradient) is not expected to further play a significant role.

Due to a pressure gradient appearing during the acoustic timescale, the speed 
of expansion, $v$, can be roughly obtained by solving:

$$
\rho \frac{\partial v}{\partial t}=\frac{\partial P}{\partial r}
$$

where $\rho$ is the material density and $P$ is the pressure. Assuming smooth variations of the physical quantities under considerations, the latter expression can be rewritten within a macroscopic description, i.e.:

$$
v=\frac{\Delta P \Delta t}{\rho \Delta r}
$$

where now $\Delta r$ corresponds to the spatial scale for which pressure variations occur, i.e. $\Delta r \simeq r_{0}$ that corresponds to the initial size of the precursor defect. In this description, $\Delta t$ is the time required for the pressure wave to travel in the precursor defect. So it corresponds to the acoustic time scale given by $\Delta t=\Delta r / c_{0}$ where $c_{0}$ is the velocity of the pressure wave. Therefore, the velocity of the expansion due to hydrodynamics reads:

$$
v=\frac{\Delta P}{\rho c_{0}}
$$

Now $\Delta P$ has to be evaluated. To do so, an equation of state is required. For the present estimation, we choose a simple Mie-Gruneisen expression: $\Delta P=\Gamma \rho \Delta e$ where $\Gamma$ is the Gruneisen coefficient and $e$ is the internal energy. The latter can be related to the absorbed power density, $W$, as: $\Delta e=W \Delta t / \rho$. The absorbed power density can be evaluated within the absorption model described in the text. Finally, one obtains:

$$
v=\frac{\Gamma W \Delta t}{\rho c_{0}}=\frac{\Gamma W r_{0}}{\rho c_{0}^{2}}
$$

Within conditions where $W \simeq 5 \times 10^{13} \mathrm{~W} / \mathrm{cm}^{3}\left(I=3 \mathrm{GW} / \mathrm{cm}^{2}\right.$, see text), with $\Gamma=2, c_{0}=5000 \mathrm{~m} / \mathrm{s}$, and $\rho=2338 \mathrm{~kg} / \mathrm{m}^{3}$, we get $v=171 \mathrm{~nm} / \mathrm{ns}$.

It is worth noting that this approach makes sense since it predicts pressure in the GPa range, in agreement with experimental observations. Otherwise, for pressure larger than the bulk modulus (standardly a few tens of GPa for optical materials), additional mechanisms should be taken into account.

\section{References}

[1] Yoreo, J. D., Burnham, A., and Whitman, P., "Developing $\mathrm{kh}_{2} \mathrm{po}_{4}$ and $\mathrm{kd}_{2} \mathrm{po}_{4}$ crystals for the world's most powerful laser," Int. Mater. Rev. 47, 113-152 (2002).

[2] Feit, M. and Rubenchik, A., "Implications of nanoabsorber initiators for damage probability curves, pulselength scaling and laser conditioning," Proc. SPIE 5273, 74-82 (2004).

[3] Hopper, R. and Uhlmann, D., "Mechanism of inclusion damage in laser glass," J. Appl. Phys. 41, 4023-4037 (1970).

[4] Walker, T., Guenther, A., and Nielsen, P., "Pulsed laser-induced damage to thin-film optical coatings-part ii: Theory," IEEE Quant. Electronics 17, 20532065 (1981).

[5] Dyan, A., Enguehard, F., Lallich, S., Piombini, H., and Duchateau, G. J. Opt. Soc. Am. B 25, 1087 (2008).

[6] Duchateau, G. Opt. Express 17, 10434-10456 (2009).

[7] Stuart, B. and et al., "Nanosecond-to-femtosecond laser-induced breakdown in dielectrics," Phys. Rev. B 53, 1749-1761 (1996). 
[8] Duchateau, G., Geoffroy, G., Dyan, A., Piombini, H., and Guizard, S., "Accepted for publication," Phys. Rev. B 83, 075114 (2011).

[9] Anisimov, S. and Makshantsev, B., "Role of absorbing inclusions in the optical breakdown of transparent media," Sov. Phys. Solid State 15, 743-745 (1973).

[10] Feit, M. and et al, "Modeling of laser damage initiated by surface contamination," Proc. SPIE 2966, 417-424 (1997).

[11] Rubenchik, A. and Feit, M., "Initiation, growth and mitigation of uv laser induced damage in fused silica," Proc. SPIE 4679, 79-94 (2002).

[12] Danileiko, Y., Manekov, A., and Nechitailo, V., "The role of absorbing defects in the laser damage of transparent materials," NBS Spec. Pub. 620 (1981).

[13] Zhia-Lin, X. and et al, "Development in laser induced extrinsic absorption damage mechanism of dielectric films," Chin. Phys. Lett. 23, 2179 (2006).

[14] Bude, J. and et al, "Effect of lattice temperature on surface damage in fused silica optics," Proc. SPIE 6720, 672009 (2007).

[15] Sugiura, H., Kondo, K., and Sawaoka, A., "Dynamic response of fused quartz in the permanent densification region," J. Appl. Phys. 52, 3375-3382 (1981).

[16] Wong, J. and et al, "Morphology and microstructure in fused silica induced by high fluence uv $3 \mathrm{w}(355 \mathrm{~nm})$ laser pulses," J. Non-cryst. Solids 352, 255-272 (2006).

[17] Kubota, A. and et al, "Densification of fused silica due to shock waves and its implications for $351 \mathrm{~nm}$ laser induced damage," Opt. Express 8, 611-616 (2001).

[18] Saito, K. and Ikushima, A. J., "Absorption edge in silica glass," Phys. Rev. $B$ 62, 8584-8587 (2000).

[19] Glezer, E., Siegal, Y., Huang, L., and Mazur, E., "Laser induced bandgap collapse in gaas," Phys. Rev. B 51, 6959-6970 (1995).

[20] Miyamoto, I., Cvecek, K., and Schmidt, M., "Evaluation of nonlinear absorptivity in internal modification of bulk glass by ultrashort laser pulses," Opt. Express 19, 10714 (2011).

[21] Sadigh, B. and et al, "First priciples calculations of the urbach tail in the optical absorption spectra of silica glass," Phys. Rev. Lett. 106, 027401 (2011).

[22] Landau, L. and Lifshitz, E., [Fluid Mechanics], Pergammon Press (1959).

[23] Rose, P., "Recent experiments in laser supported absorption waves," Acta Astronautica 2, 941-953 (1975).

[24] Zelikin, N. and et al, "Observation of absorption waves in a transparent dielectric," Sov. Tech. Phys. Lett 4, 522-523 (1978).

[25] Demos, S., Negres, R., Demange, P., and Feit, M. Opt. Express 18, 13788 (2010).

[26] Carr, C., Radousky, H., and Demos, S., "Wavelength dependence of laserinduced damage: determining the damage initiation mechanisms," Phys. Rev. Lett. 91, 127402 (2003).

[27] C.S. Liu, N. Kioussis, S. D. and Radousky, H., "Electron or hole-assisted reactions of h defects in hydrogen-bonded kdp," Phys. Rev. Lett. 91, 015505 (2003).

[28] Chirila, M., Garces, N., Halliburton, L., Demos, S., Land, T., and Radousky, H. J. Appl. Phys 94, 6456 (2003).

[29] Rethfeld, B., "Unified model for the free-electron avalanche in laser-irradiated dielectrics," Phys. Rev. Lett. 92, 187401 (2004). 
[30] Dieguez, E. and Cabrera, J. J. Phys. D: Appl. Phys. 14, 91 (1981).

[31] Carr, C., Bude, J., and DeMange, P. Phys. Rev. B 82, 184304 (2010).

[32] Duchateau, G., Hébert, D., and Hallo, L., "Modeling laser-induced damage of kdp crystals by nanosecond pulses: a preliminary hydrodynamic study," Proc. SPIE 7842, 78420S (2010).

[33] Hebert, D., Hallo, L., Voisin, L., Desanlis, T., Galtie, A., Bicrel, B., Maunier, C., Mercier, P., and Duchateau, G., "A kdp equation of state for laser-induced damage applications," J. Appl. Phys. 109, 123527 (2011).

[34] Carr, C., Feit, M., Johnson, M., and Rubenchik, A., "Complex morphology of laser-induced bulk damage in $\mathrm{kh}_{2-x} \mathrm{~d}_{2 x} \mathrm{po}_{4}$ crystals," Appl. Phys. Lett. 89, 131901 (2006).

[35] Friebele, E., Griscom, D., and Stapelbroek, M. Phys. Rev. Lett. 42, 1346 (1979).

[36] Mao, X., Mao, S., and Russo, R. Appl. Phys. Lett. 82, 697 (2003).

[37] Tzortzakis, S., Papazoglou, D., and Zergioti, I. Opt. Lett. 31, 796 (2006).

[38] Negres, R., Feit, M., and Demos, S. Opt. Express 18, 10642 (2010).

[39] Raman, R., Negres, R., and Demos, S. Opt. Eng. 50, 013602 (2011).

[40] Juodkazis, S., Nishimura, K., Tanaka, S., Misawa, H., Gamaly, E., LutherDavies, B., Hallo, L., Nicolai, P., and Tikhonchuk, V. Phys. Rev. Lett. 96, 166101 (2006). 


\section{FIGURES AND CAPTIONS}

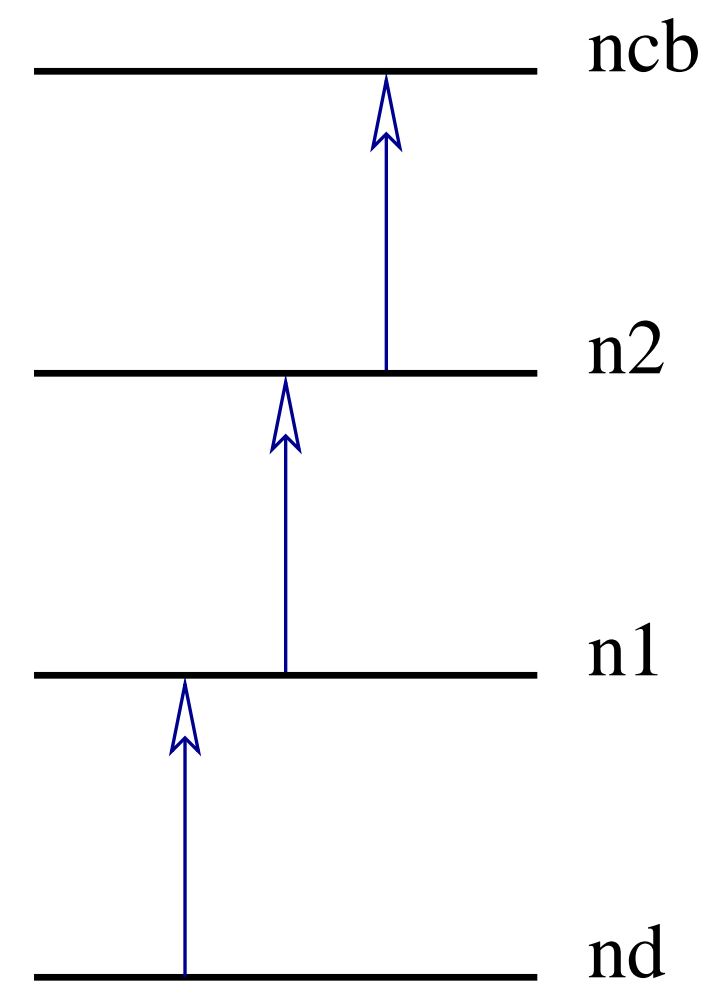

Figure 1: Schematic illustration of the band structure and of the sequential one-photon absorption at $3 \omega$. 

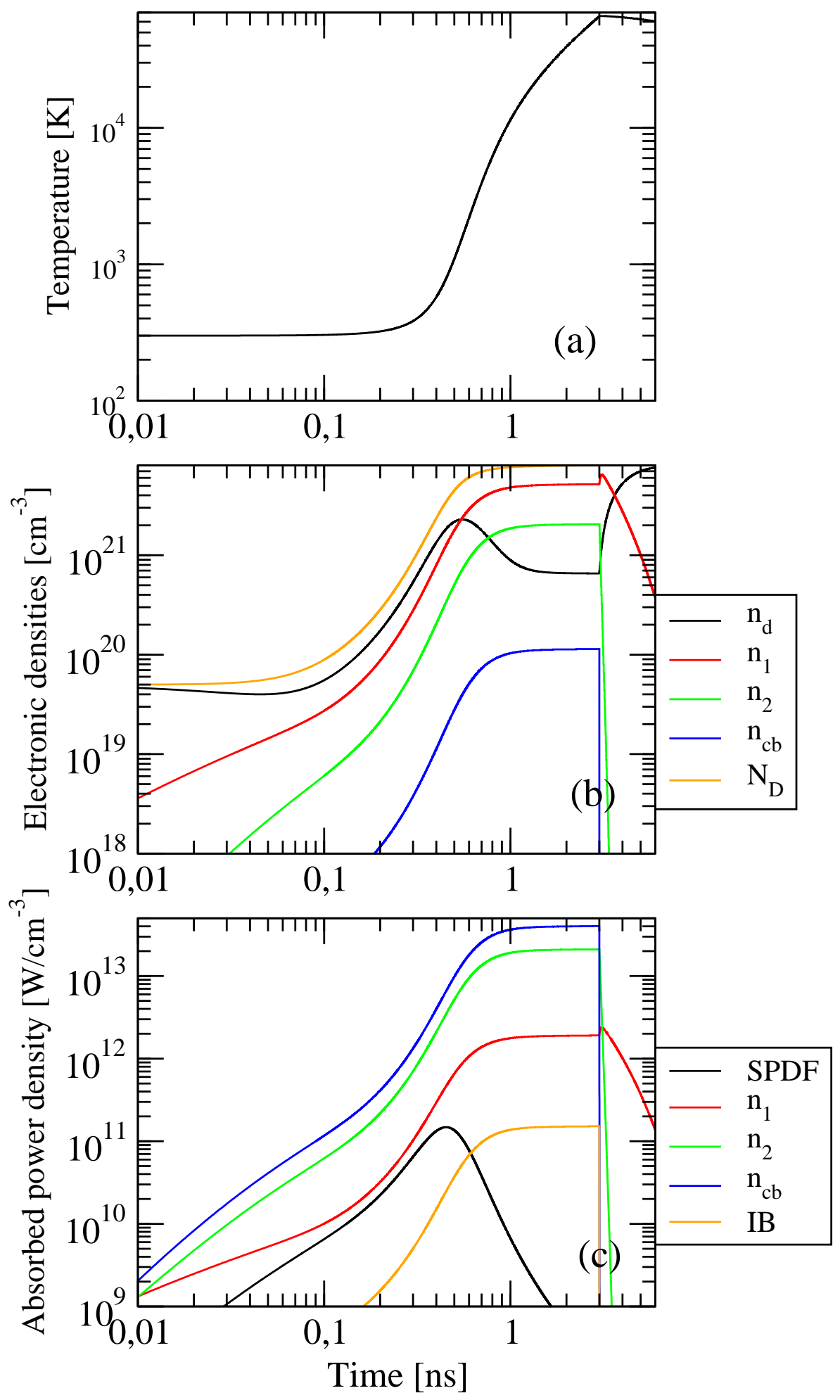

Figure 2: Evolution of (a) the temperature, (b) electronic densities, and (c) absorbed power density as a function of time at the center of the precursor defect. The legends for each color-coded profile are provided in the inset. 

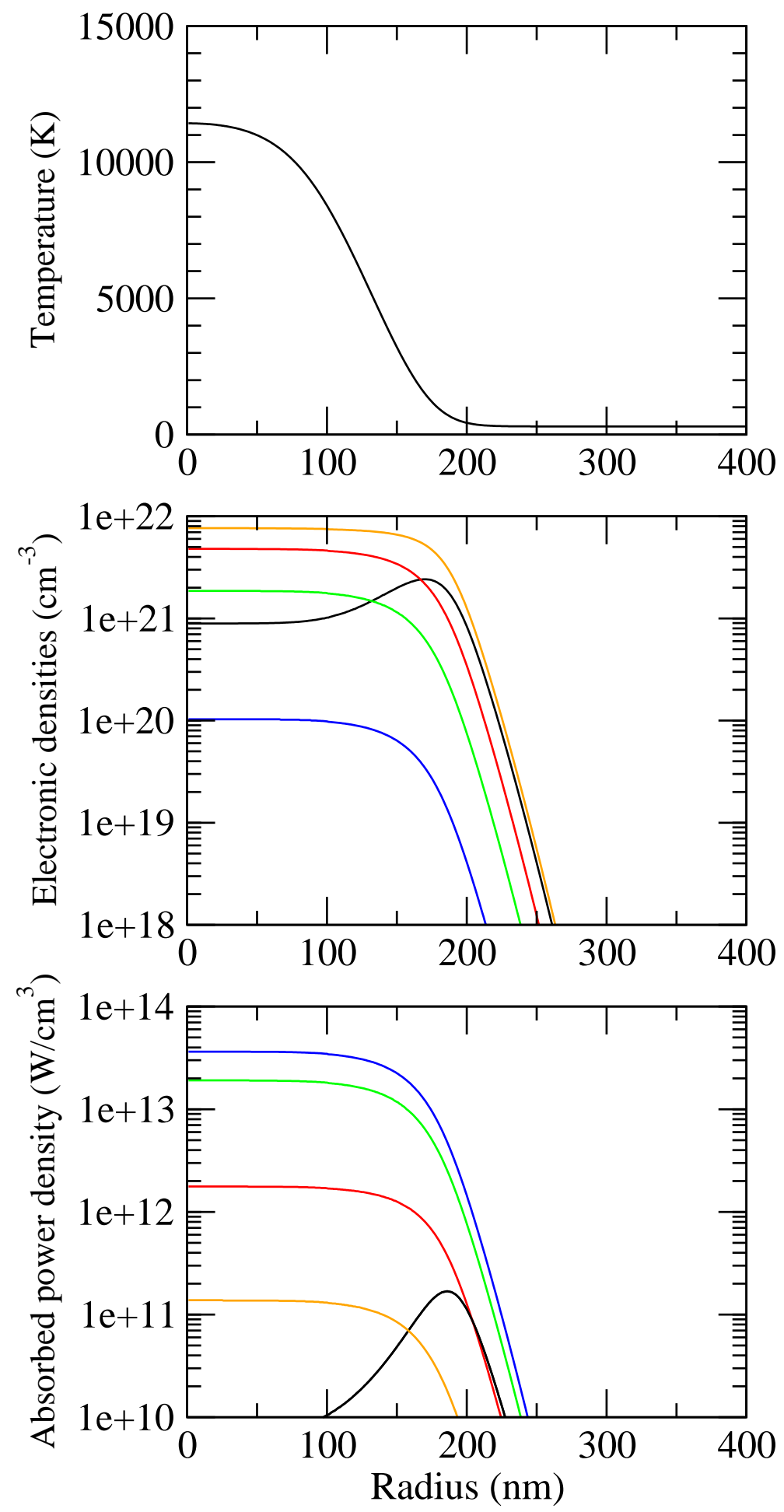

Figure 3: Evolution of (a) the temperature, (b) electronic densities, and (c) absorbed power density as a function of the radius at $1 \mathrm{~ns}$ delay. The legend of each color-coded profile is the same as in Fig. 2. 
"Temperature"

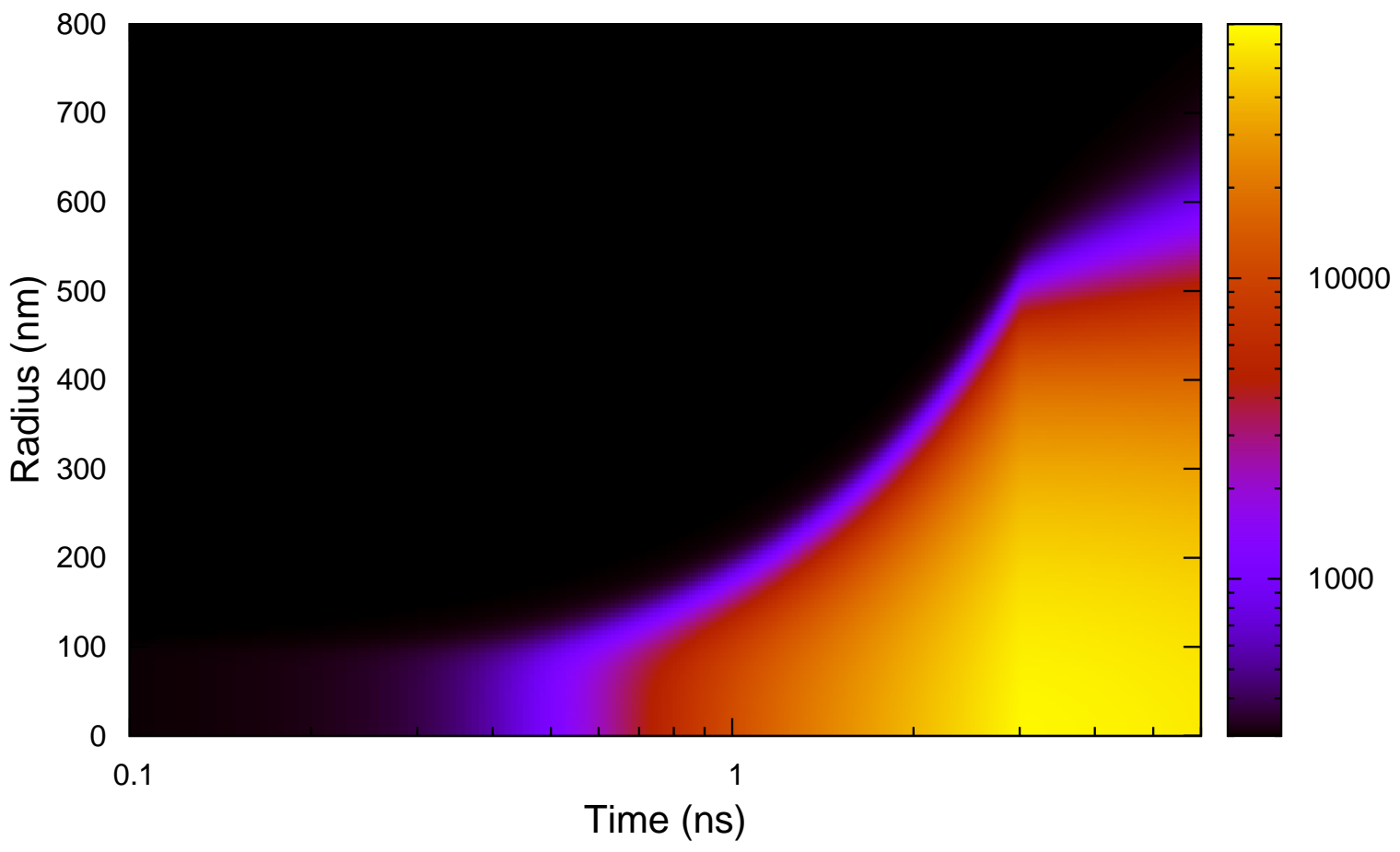

Figure 4: Temperature (in K, logarithmic scale) as a function of space and time. 


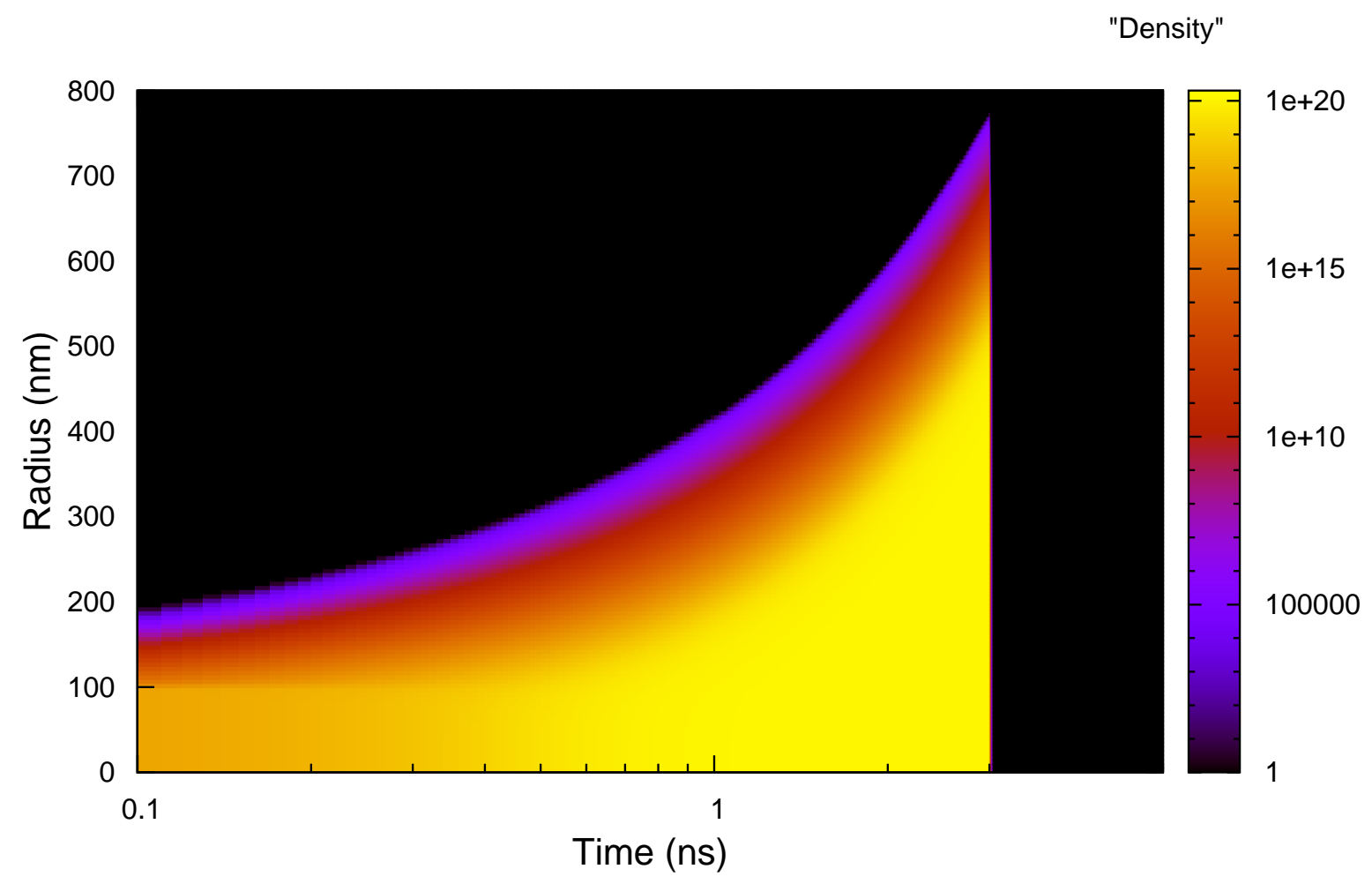

Figure 5: Density of conduction electrons (in $\mathrm{cm}^{-3}$, logarithmic scale) as a function of space and time. 


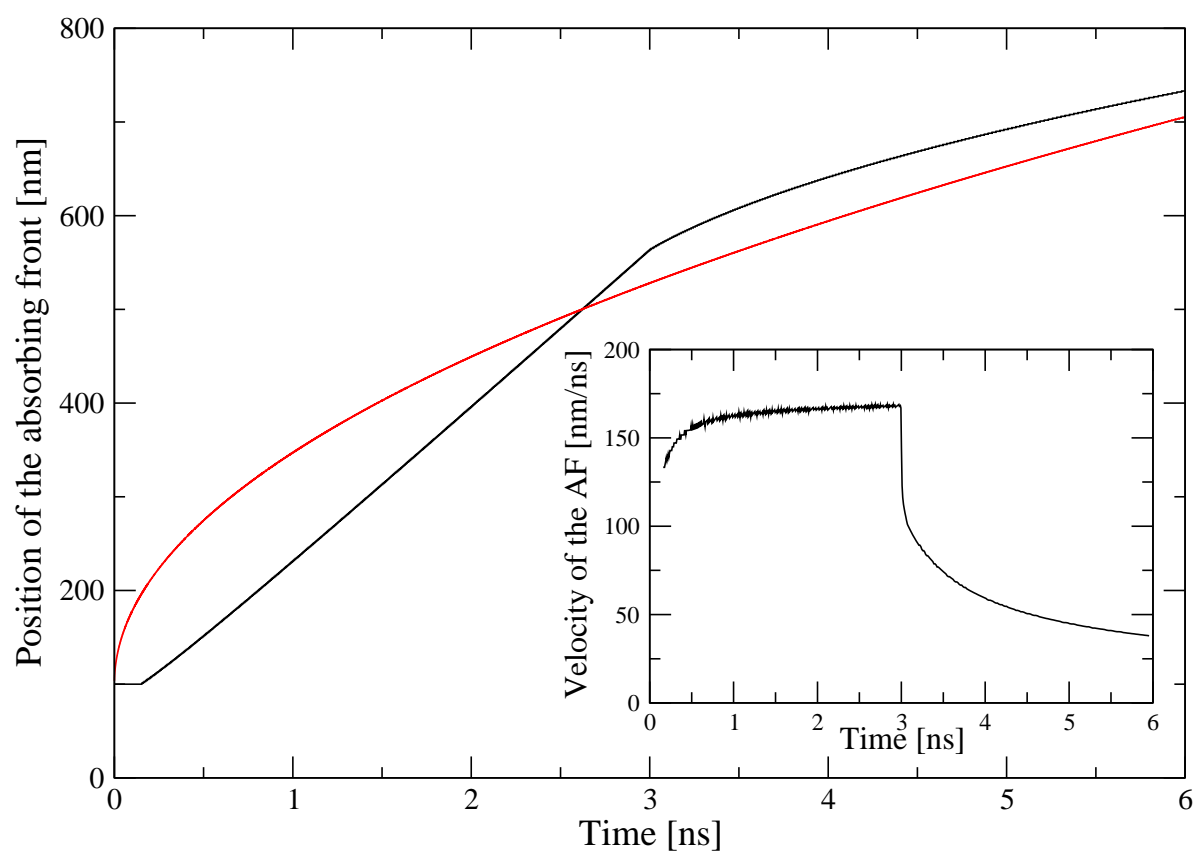

Figure 6: Evolution of the position of the absorbing front as a function of time (black curve). It is defined such that $N_{d} \geq n_{0}$, see text for more detail. The red curve is a fit of the latter curve with the function $y=2 \sqrt{D t}$ where $D$ is the fitting parameter whose optimized value is $D=15000 \mathrm{~nm}^{2} / \mathrm{ns}$.

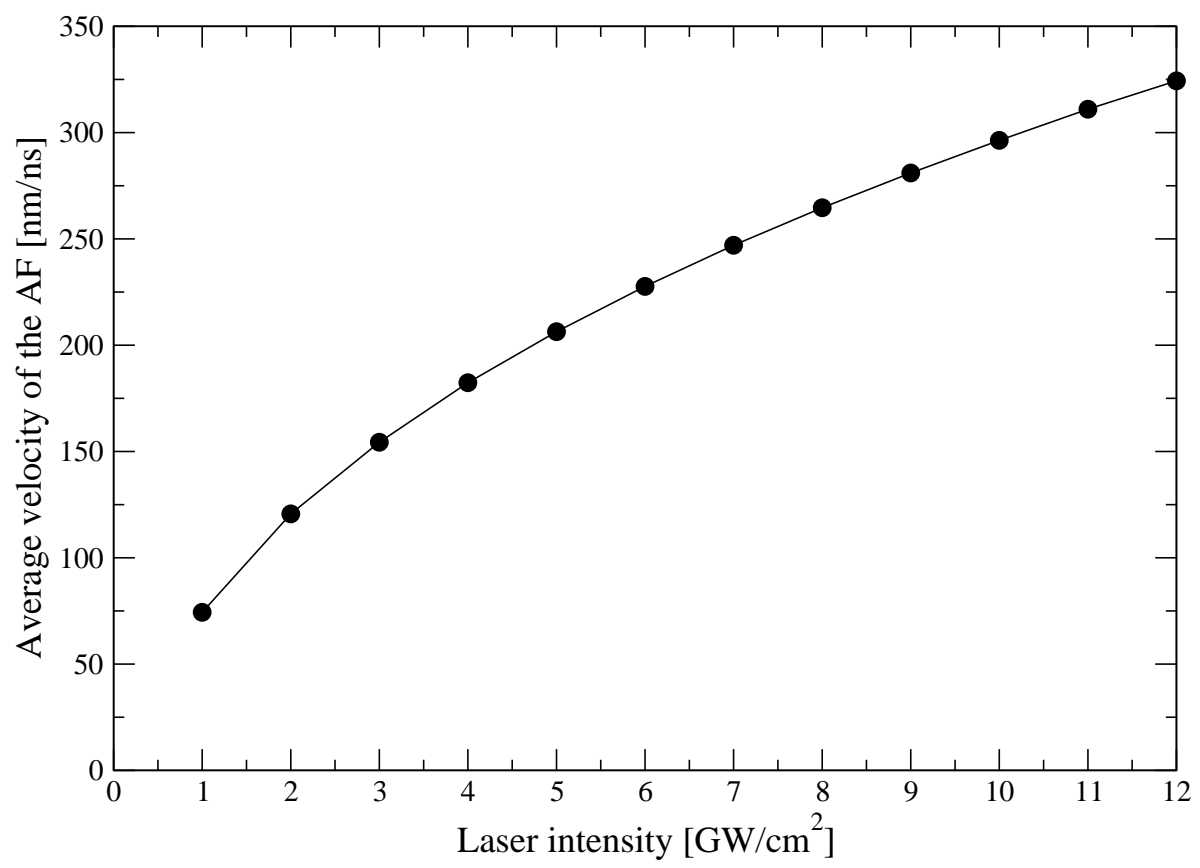

Figure 7: Evolution of the average velocity of the absorbing front as a function of the laser intensity. 

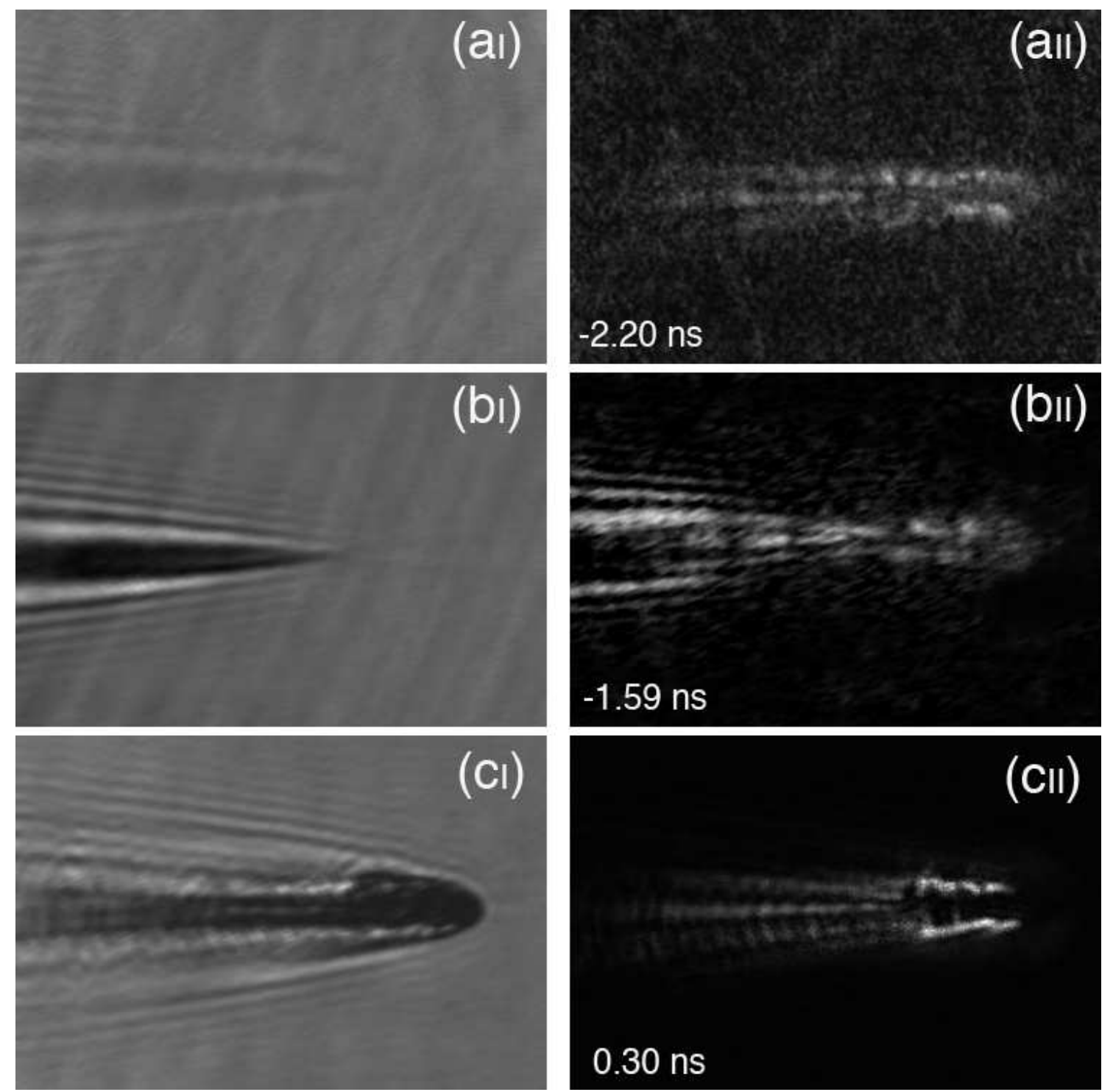

Figure 8: Transient microscopic images $(150 \mu m \times 100 \mu m)$ during laser damage initiation with a focused beam in the bulk of fused silica showing the parallel (I) and orthogonal (II) image components at delays a) $-2.20 \mathrm{~ns}, \mathrm{~b}$ ) $-1.59 \mathrm{~ns}$ and, c) $0.30 \mathrm{~ns}$. The delays are measured between the peak intensities of the pump and probe pulses. 